\title{
IMPACT OF THE VIRTUAL REALITY MIRROR IN INDIAN RETAIL STORES
}

\author{
Aditya Iyer ${ }^{1}$ \\ ${ }^{1}$ Symbiosis Institute of Business Management - Bengaluru \\ Symbiosis International University
}

\begin{abstract}
The research paper focuses on the impact of Virtual Reality Mirror on the Indian retail store, which is dependent on functions such as user experience, purchasing decision and demographic variables. The user experience is dependent on the immersive experience like VR mirror and the average time spent for the trial of clothes. The purchasing decision is dependent on look and feel of fabric, long queues in stores and average shopping time. The demographic variables used in the research are age and gender. A qualitative interview with an experiential store manager was conducted to get insights about the impact of immersive technologies on the retail industry. A survey was also conducted to gather the abovementioned variables and trends were plotted graphically which depicted demographic sectors that can be targeted by retail stores to increase sales.
\end{abstract}

Keyword: Virtual Reality, VR Mirror, User Experience, Retail Stores

\section{Introduction}

Virtual reality (VR) is the use of a simulated environment, which is a present virtual environment that allows a person to interact with three-dimensional (3D) sensory environments. VR applications enable the user to interact in a virtual environment created by a computer that simulates by using interactive devices in the form of headgear, glass framers, goggles or bodysuits. The typical VR user wears a helmet with a stereoscopic view of the animated images in a simulated environment. The telepresence is affected by motion sensors that pick the user's movements and adjust the view on the screen accordingly, usually real-time streaming. Thus, one can get a tour of a simulated environment, experiencing changing viewpoints and perspectives that are convincingly related to one's body motions. For example, gloves that allow the flow of data to and fro are equipped with a force-feedback mechanism that provides a sensation of touch. A user can pick up the objects and manipulate these objects seen in his virtual reality environment.

Reports today indicate that for the first time, the sales of VR Mounted Displays especially head mounted displays has crossed 1 million in one quarter; one can expect the value of such devices sold to rise from $\$ 1.5$ billion USD in 2017 to $\$ 9.1$ billion USD by the end of 2021 . It is vital to note that younger generations ( $\mathrm{Y}$ and $\mathrm{Z})$ are the ones who are most interested in VR technology. Upcoming releases of standalone VR head-mounted devices like Occulus Go, HTC Vive Focus, together with the declining costs of these devices due to economies of scale, can hugely increase the future usage of VR. In addition to this, according to Gartner's top 10 strategic trends, Augmented Reality (AR) and Mixed Reality (MR) are ranked among the 10 strategic trends for 2018 .

\section{RESEARCH OBJECTIVE}

To study the impact of Virtual Reality Mirror in Indian Retail Store

\section{RESEARCH PROBLEM}

The emergence of online stores has resulted in lesser footfall in physical retail stores. Thereby there is a need to have technological innovations to enhance the shopping experience at retail stores in order to increase the footfall and drive sales.

Businesses today are investing hugely in advanced technologies like VR, AR but are unable to deploy them effectively due to lack of evidence towards the impact of these technologies on Retail Stores.

\section{LITERATURE REVIEW}

A paper by (Park, M., Im, H., \& Kim, D. Y. (2018). "Feasibility and user experience of virtual reality fashion stores") describes that there is a limited amount of research used a truly immersive system to examine the difficulties and benefits of using it. Developing an immersive VR environment that simulates a real shopping experiences with users' movements is critical in enriching users' shopping experiences as well as understanding in-store consumer behavior. A content analysis was conducted to review the method, variables, contexts, and devices used in the previous studies. A total of 40 females (mean age $=21.05$ ) participated in the study. The current study provided a content analysis of previous studies on VR usage for retail and filled the gap in the literature by developing VR apparel stores and empirically testing them using a truly immersive VR system. The overview and findings provide important implications to practitioners and scholars that immersive VR can be a promising tool to use even though there are a few challenges. The VR system used in the study did not allow users to touch 
or move virtual products, limiting realism of the VR environment.

Whether and how VFR influences sales and post-sales outcomes based on the limited literature, and retailers hesitate in adopting the technology due to concerns about its profit prospects. Two large-scale field experiments (Study 1 and Study 2) with real-world behavioral data (e.g., actual purchases and product returns) to test our hypotheses, and a controlled lab experiment (Study 3) using self-reported measures of user perceptions to delineate the theoretical mechanisms behind the effects observed in the field experiments.

Study 1 at an online retailer of young women's apparel and analyzed a total of 933 product items and 24,435 product purchases during the observation period. Although the introduction of VFR can cause an increase in sales, its effectiveness varies significantly depending on the types of avatars employed and the simultaneous presence of other promotional venue. Specifically, VFR with personalized avatars can lead to a greater increase in sales than nonpersonalized avatars, but only when CVD is unavailable. Second, VFR helps increase post-purchase customer satisfaction and reduce the rate of product returns, and personalized VFR is more effective on these two outcome variables than non-personalized VFR. The lab experiment reveals the theoretical mechanisms behind the effects observed from the field experiments: the positive effect of personalized VFR on purchases can be attributed to reduced perceived risk and increased enjoyment; however, when CVD is present, personalized VFR increases users' self-discrepancy, which in turn reduces the likelihood of purchase. The research is confined to analysis of women but not males and is focused on high end markets.

According to (Shuai Yang, Guiyang Xiong 2019), whether and how VFR influences sales and post-sales outcomes based on the limited literature, and retailers hesitate in adopting the technology due to concerns about its profit prospects. Two large-scale field experiments (Study 1 and Study 2) with realworld behavioral data (e.g., actual purchases and product returns) to test our hypotheses, and a controlled lab experiment (Study 3) using self-reported measures of user perceptions to delineate the theoretical mechanisms behind the effects observed in the field experiments. Study 1 at an online retailer of young women's apparel and analyzed a total of 933 product items and 24,435 product purchases during the observation period.

Although the introduction of VFR can cause an increase in sales, its effectiveness varies significantly depending on the types of avatars employed and the simultaneous presence of other promotional venue. Specifically, VFR with personalized avatars can lead to a greater increase in sales than nonpersonalized avatars, but only when CVD is unavailable. Second, VFR helps increase post-purchase customer satisfaction and reduce the rate of product returns, and personalized VFR is more effective on these two outcome variables than non-personalized VFR. The lab experiment reveals the theoretical mechanisms behind the effects observed from the field experiments: the positive effect of personalized VFR on purchases can be attributed to reduced perceived risk and increased enjoyment; however, when CVD is present, personalized VFR increases users' self-discrepancy, which in turn reduces the likelihood of purchase. The reserach is confined to analysis of women but not males and is focused on high end markets.

"Interactive Digital Mirror" published in 2016 describes how to segment objects from the input video stream, how to process the segmented objects scene, modifying them, and how to realistically render the new scene are the main challenges of this project. Techniques used are Face detector, IR Illumination, Mobile-Tablet Application, Computer Vision. The solutions drawn were that face detecting digital mirror is developed. Different than regular mirrors, digital mirrors can change the image of the scene reflected on the mirror, but leaving most of the scene, or at least part of it, looking the same. This research article is limited to process faces and does not give image of the full body.

But according to (Yang, S., \& Xiong, G. (2019). Try It On! Contingency Effects of Virtual Fitting Rooms), there is a lack of clarity over whether, how and when VFR influences product sales. The question is of utmost importance to managers because of the considerable cost of implementation and maintenance. Further, extant research has rarely examined how the virtual reality technology influences post-purchase metrics. This study empirically tests the causal effects of different VFR designs on consumer purchases and postpurchase behaviors using large-scale field experiments and unveil the underlying theoretical mechanisms. This paper conducted two large-scale field experiments (Study 1 and Study 2) with real-world behavioral data (e.g., actual purchases and product returns) to test the hypotheses, and a controlled lab experiment (Study 3) using self-reported measures of user perceptions to delineate the theoretical mechanisms behind the effects observed in the field experiments. Insights from field experiments can be readily applied to managerial decision making because they demonstrate the economic magnitude of the effect, while lab experiment is powerful to reveal the psychological process that would otherwise be difficult to measure in real-world settings. 


\section{PROPOSED MODEL}

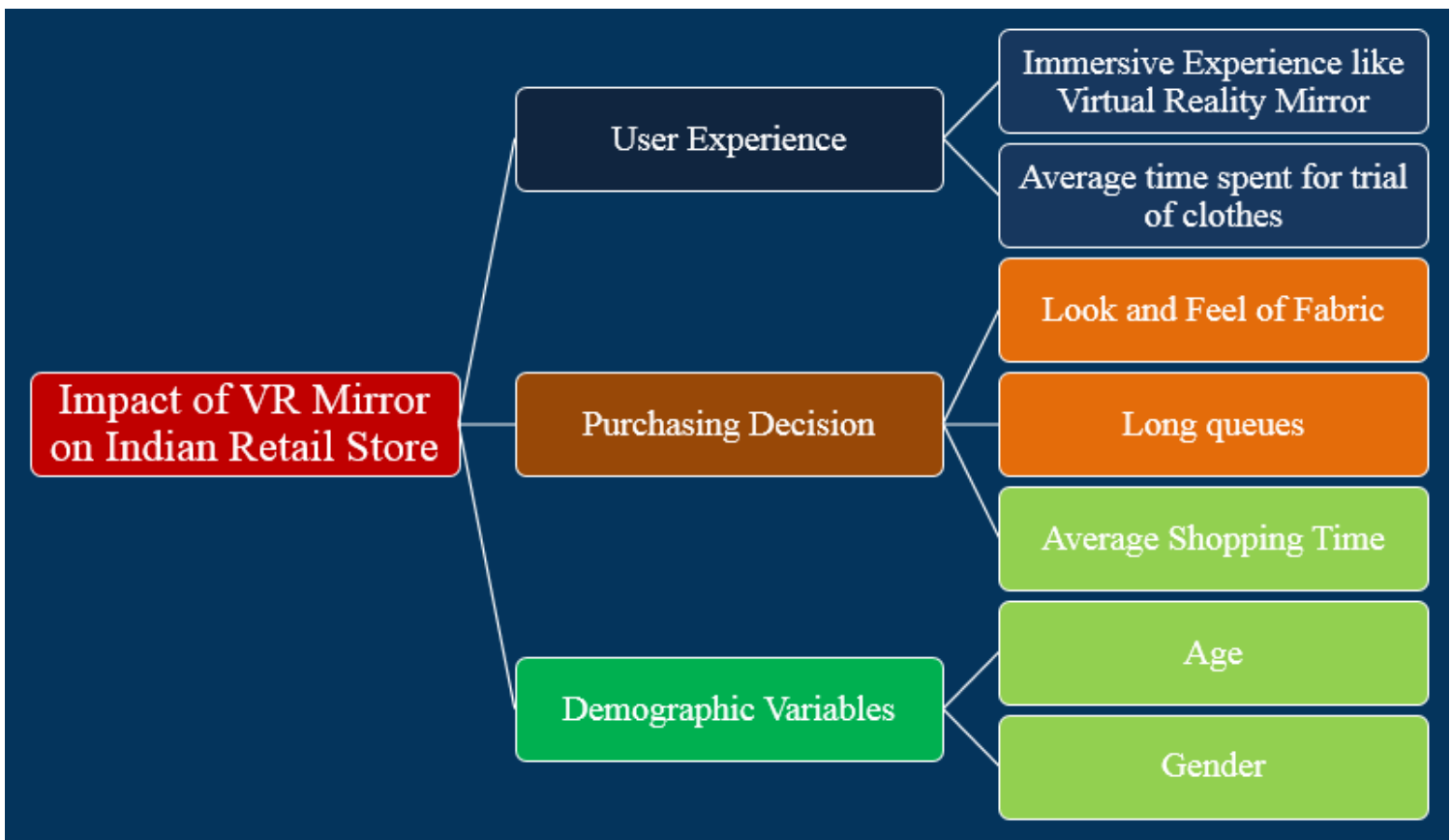

\section{METHODOLOGY}

Types of methods used for Research.

1) Qualitative Interviews

2) Sentiment Analysis

3) Questionnaire

\section{Qualitative Interview at Experiential Store}

I conducted a qualitative interview at the Puma Experience outlet with the store manager. The interview gave me insights about various topics like Virtual Reality, Customer Preferences, Market Perception about virtual reality etc. During the meeting, I got to know how Puma is using virtual reality to drive its sales. The manager went on to say that there is no direct connection between the technology and the sales figures; however, technology undoubtedly augments the shopping experience of the customer. Apart from that various virtual reality kiosks which have been set up at the store also increase the walk-ins for the store.
Figure 1: The model proposed

Overall, I could understand that there is a positive mindset of retail stores towards virtual reality and that this piece of technology can surely attract customers and also augment their buying experience at the same time.

\section{Sentiment Analysis}

I analysed various tweets and comments made on social media regarding the use of virtual reality for shopping purposes and I did a sentiment analysis on the sample data which I gathered. I could find out that there is a very positive outlook towards the use of virtual reality to augment the shopping experience. This goes on to say that the customers are looking forward to having this kind of technology in the retail stores, and the companies should implement this kind of solutions to deliver an immersive shopping experience to its customers. 


\begin{tabular}{|c|c|c|c|c|}
\hline $\boldsymbol{\wedge}$ & negative & positive & sentiment & file \\
\hline 1 & 5 & 40 & 35 & 3 Ways Virtual Reality Will Transform eCommerce.txt \\
\hline 2 & 9 & 29 & 20 & 5 Ways Augmented Reality (AR) Is Transforming Retail.txt \\
\hline 3 & 2 & 31 & 29 & 7 Examples of Retailers Making Use of Augmented Reality.txt \\
\hline 4 & 7 & 32 & 25 & AR Retail Trends Disrupting the Industry.txt \\
\hline 5 & 11 & 15 & 4 & Are retailers ready for shift to virtual reality shopping.txt \\
\hline 6 & 36 & 88 & 52 & AUGMENTED REALITY RETAIL APPLICATIONS \& VR STORE S... \\
\hline 7 & 9 & 104 & 95 & How Augmented Reality Benefits Retail and Shopping.txt \\
\hline 8 & 11 & 35 & 24 & HOW AUGMENTED REALITY IS TRANSFORMING RETAIL.txt \\
\hline 9 & 7 & 44 & 37 & How These Retailers Use Augmented Reality to Enhance the... \\
\hline 10 & 6 & 52 & 46 & Retailers Have A Lot To Gain From AR and VR.txt \\
\hline 11 & 9 & 23 & 14 & Shopping in Virtual Reality Stores.txt \\
\hline 12 & 8 & 60 & 52 & The Future of Retail is Virtual.txt \\
\hline 13 & 22 & 86 & 64 & The Future of Virtual Reality in Retail.txt \\
\hline 14 & 12 & 60 & 48 & Virtual Reality in Retail.txt \\
\hline 15 & 14 & 30 & 16 & VR in Retail-The Future of Shopping Is Virtual and Augment... \\
\hline 16 & 16 & 29 & 13 & What is the Future of Augmented and Virtual Reality.txt \\
\hline
\end{tabular}

Figure 2: Sentiment Analysis

\section{Word Cloud}

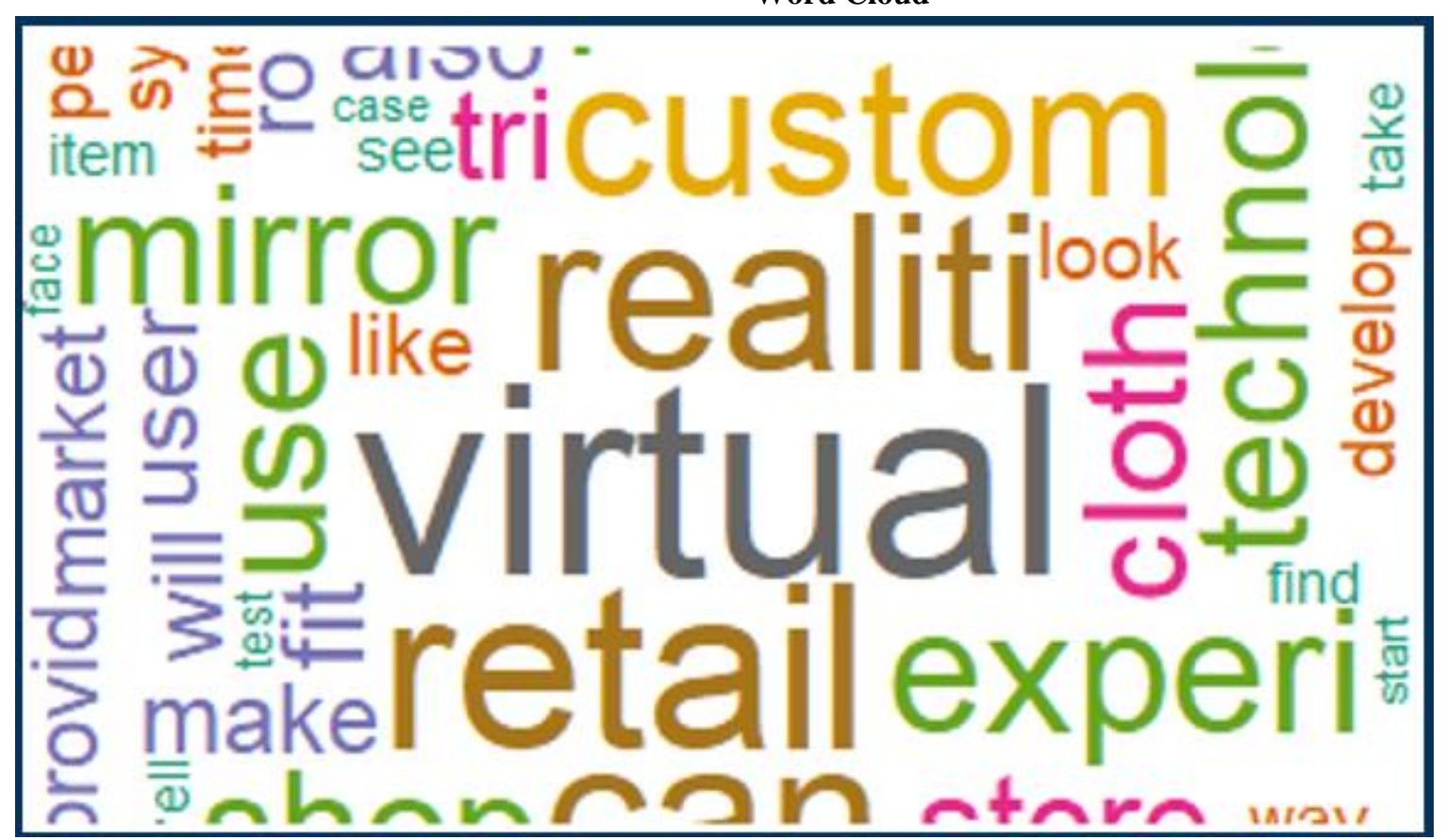

Figure 2: Generated Word Cloud 


\section{Analysis of Floated Questionnaire}

To have a further understanding regarding the customer preferences, I floated out a questionnaire to a target audience lying within the age group of 20-50 years. The survey was explicitly targeted towards middle-class people who would be working in corporates since they would be the ones who would be shopping in this kind of retail stores.

Using the questionnaire, I was able to get 109 responses, which gave me a more in-depth understanding of what people are expecting from retail outlets.

\section{What is your age?}

109 responses

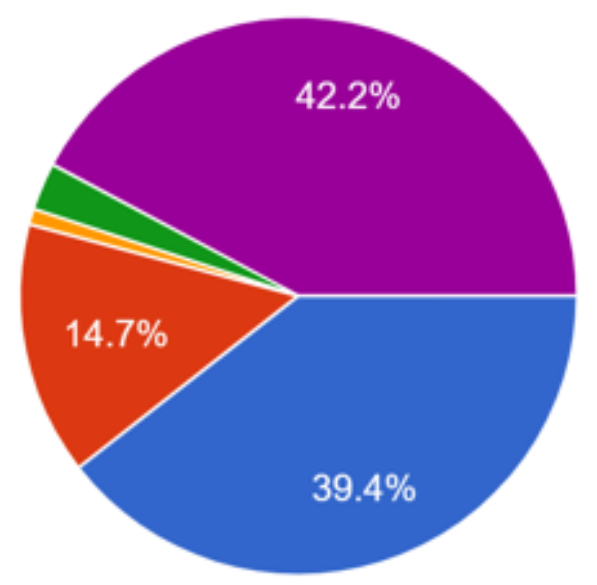

Figure 3: Chart for the age of respondents

\section{Count of What is the average time you spend while shopping in retail outlets?}

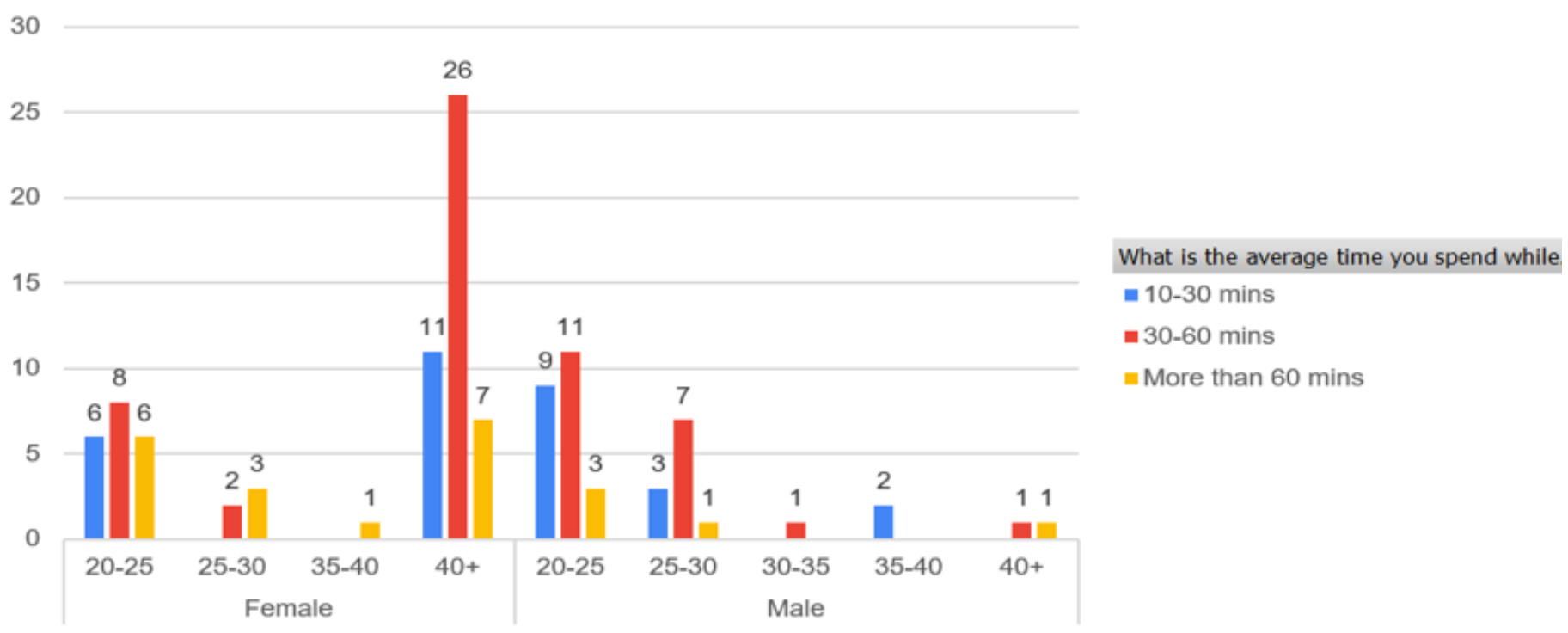

Gender - What is your age? -

Figure 4: Graph for average time spent while shopping in retail outlets 


\section{Count of What is the average time you spend trying out different outfits while shopping?}
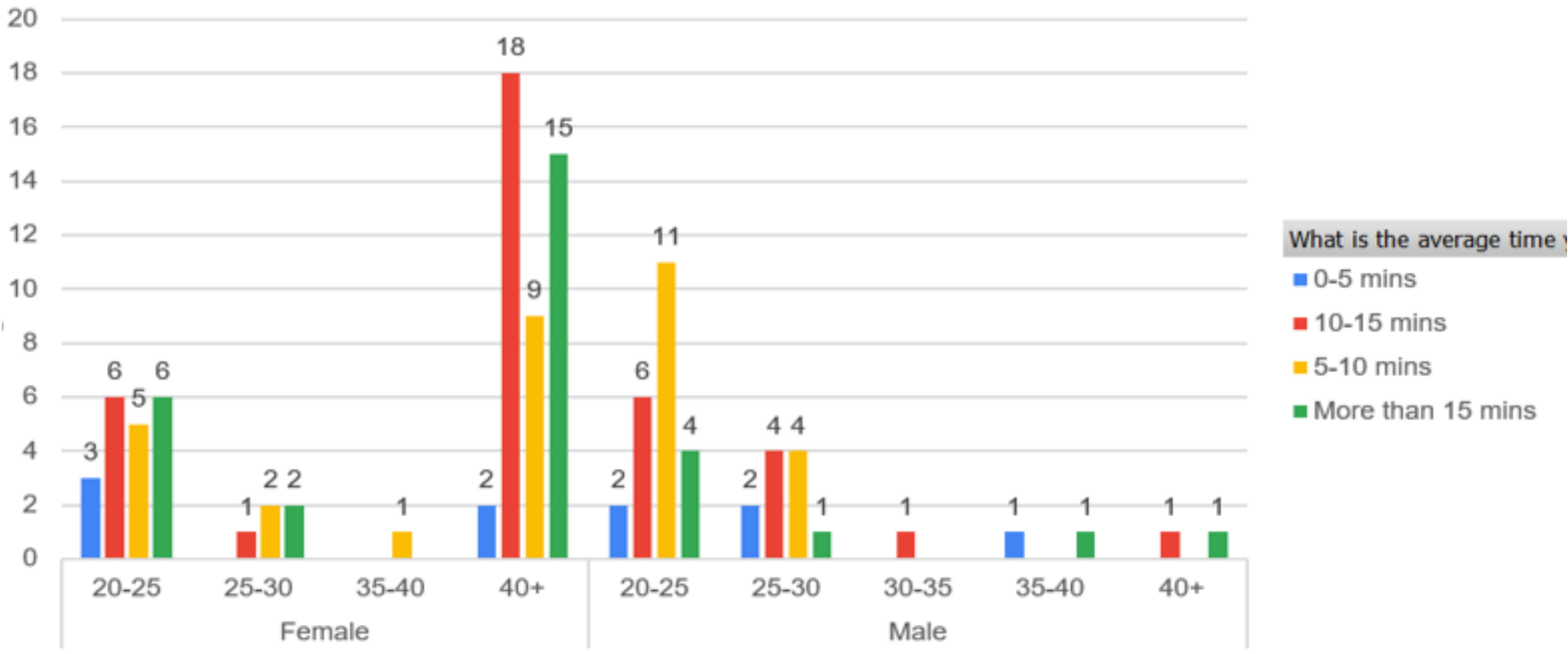

Gender - What is your age? -

Figure 5: Graph of average time spent for trying out different outfits while shopping

\section{Count of Do you think the long queues impact the purchasing decisions of the customers?}

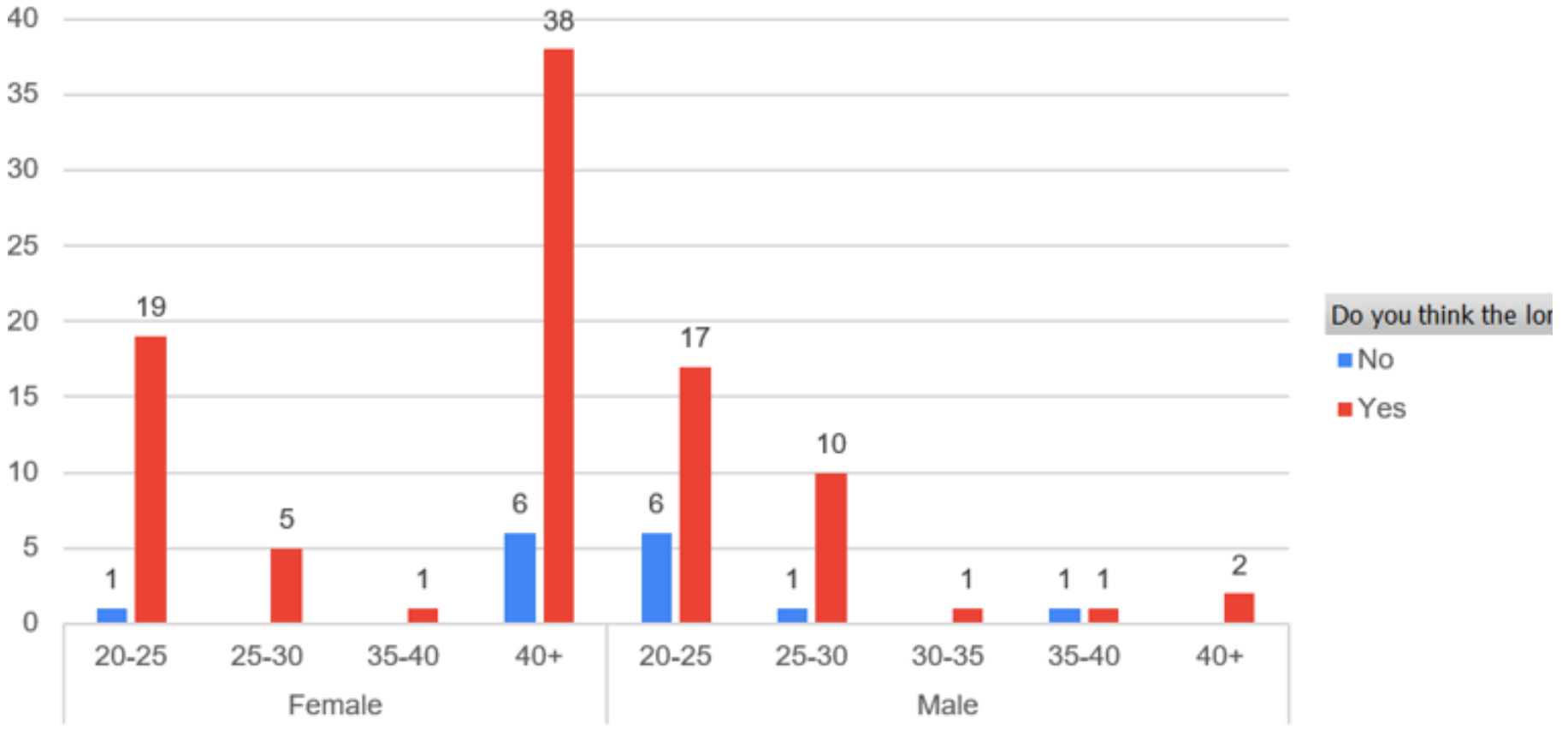

Figure 6: Graph for long queues impacting the purchase decisions 


\section{Count of What is the average time you spend while shopping in retail outlets?}

30

25

20

15

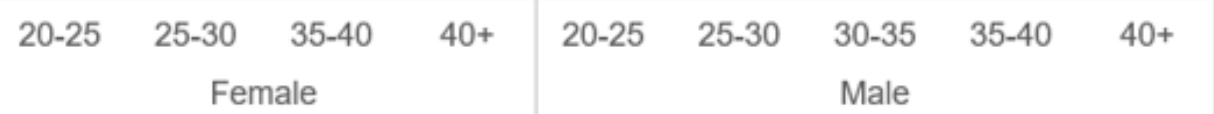

What is the average time you spend while -10-30 mins

=30-60 mins

more than 60 mins

Figure 7: Graph for gender vice Average time spent while shopping in retail outlets Count of As a customer would you prefer a physical fitting room over a virtual realty mirror to try out the outfits?

45

$40-39$

35

30

25

20

15

10

0

0

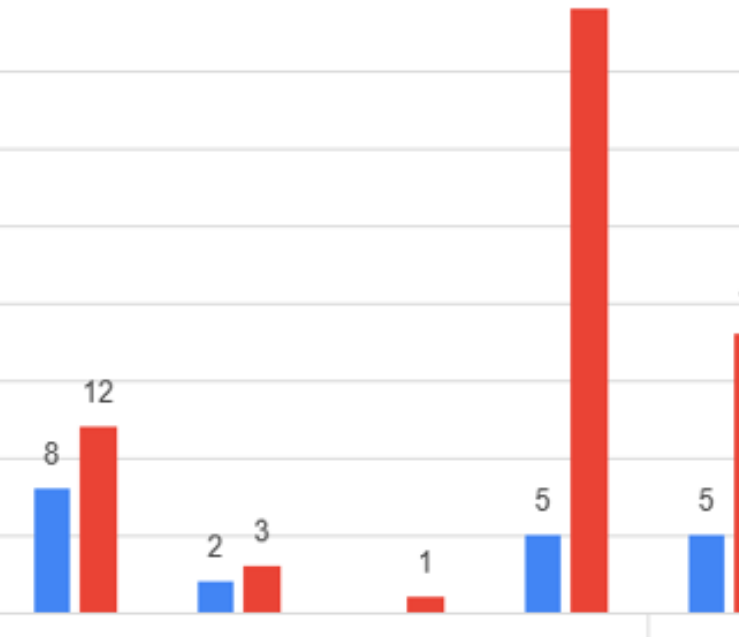

20-25

25-30

$35-40$

$40+$

20-25

18

As a customer would

- No

-Yes

Female
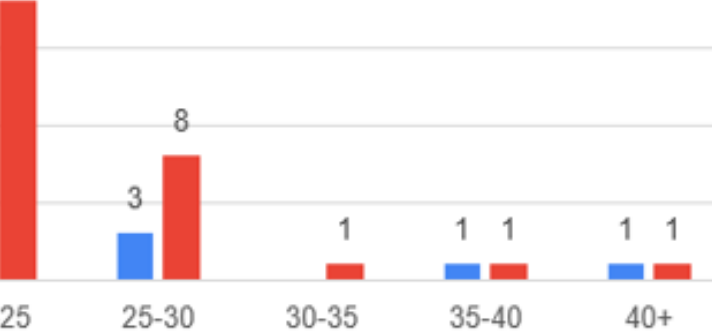

Figure 8: Graph for Preference of Physical Fitting Room over VR Mirror 


\section{Count of Do you prefer immersive experience like virtual reality mirrors etc over physical trials?}

30

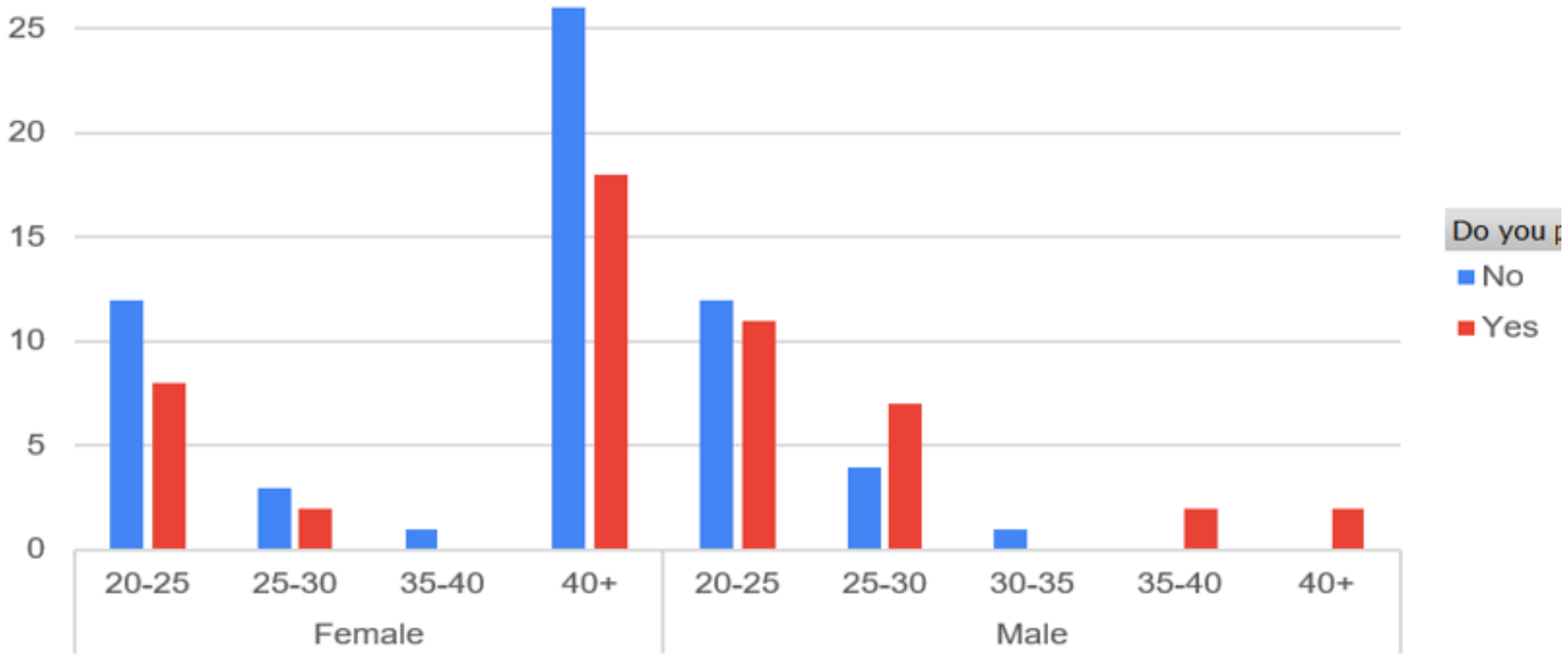

Figure 9: Graph for Preference of VR Mirror over Physical Trials

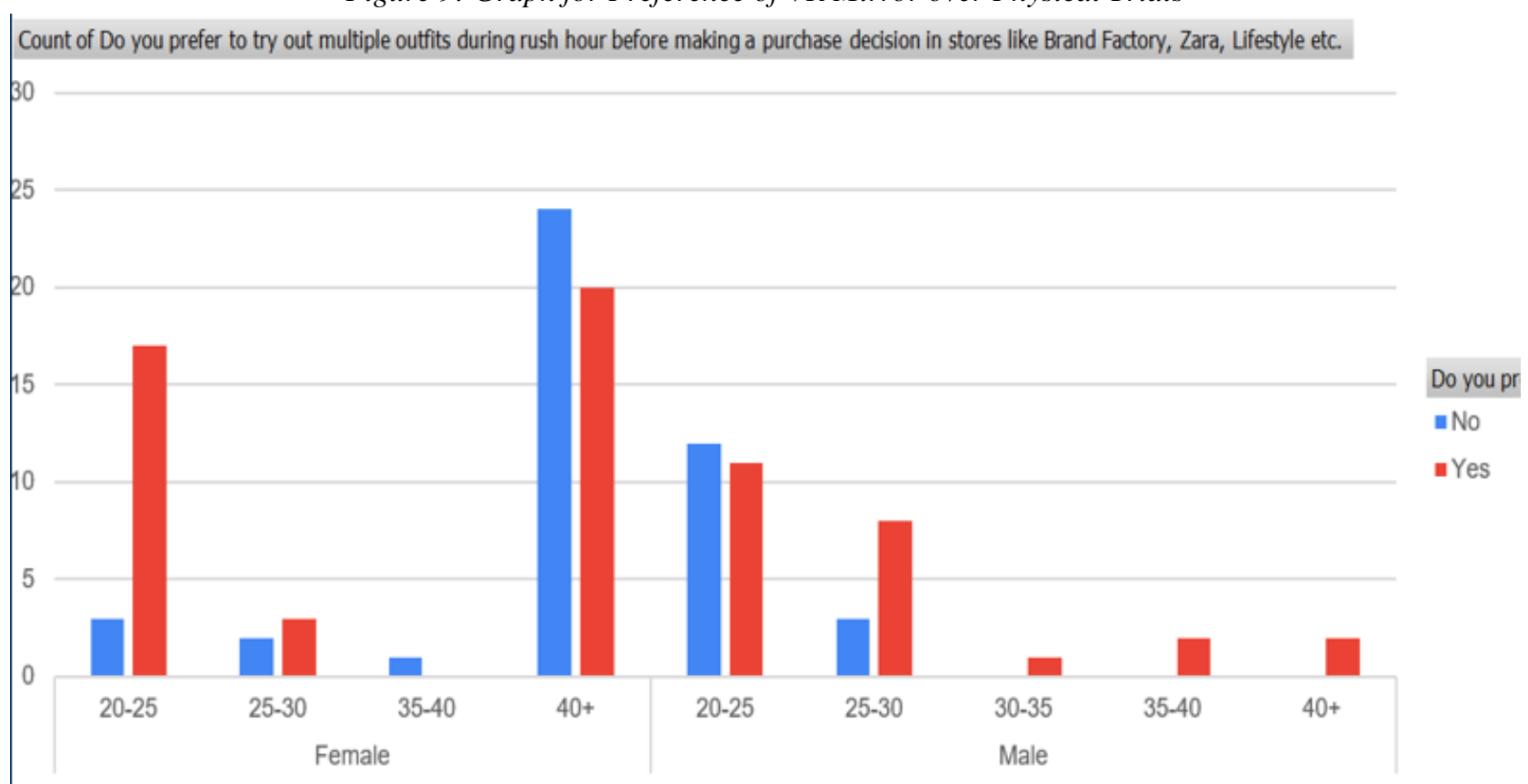

Figure 10: Graph for the impact of rush hour on purchase decision

\section{LIMITATIONS AND SCOPE}

1. The research only includes people who fall in the age group of 20-50 years.

2. The sample size used for analysis is quite limited in nature. With a sample size of 109 respondents, It is difficult to arrive at concrete conclusions which can be used to make managerial decisions in retail outlets.

3. The research is limited only to apparels.

4. The scope of research is limited to multi-brand retail stores.

5. The scope of research does not apply to luxury retail stores.

\section{CONCLUSION AND FUTURE SCOPE}

The research paper tried to understand whether immersive tech like Virtual Reality Mirrors can enhance the shopping experience of the shoppers and thereby look at a possibility of increasing the sales in the retail outlets. We could see that people prefer to have these kinds of technologies present in the store to augment their shopping experience. 
After conducting qualitative research, I have formulated a hypothesis which could be used for further quantitative research.

Hypothesis: Advance technologies like VR have an impact on sales in retail stores

I have also formulated a multi-linear regression model, which can be further used for quantitative analysis.

$\mathrm{Y}=\mathrm{b} 0+\mathrm{b} 1 \times 1+\mathrm{b} 2 \times 2+\mathrm{b} 3 \times 3$

Where $\mathrm{y}=$ sales (dependent variable)

$\mathrm{x} 1$ = Average shopping time (independent variable)

$\mathrm{x} 2=$ Virtual reality mirror (independent variable)

$\mathrm{x} 3$ = Age group (independent variable)

In order to arrive at concrete conclusions which can lead us to believe that virtual reality mirrors can increase sales in retail outlets, the above model can be used for further quantitative analysis.

\section{References:}

[1] Yang, S., \& Xiong, G. (2019). Try It On! Contingency Effects of Virtual Fitting Rooms. Journal of Management Information Systems, 36(3), 789-822.

[2] Park, M., Im, H., \& Kim, D. Y. (2018). Feasibility and user experience of virtual reality fashion stores. Fashion and Textiles, 5(1), 32.

[3] Sourabh, B., Darshan, C., Hemant, D., Priyanka, G., \& JP, R. (2017). Implementation of Virtual Dressing Room using Newton's Mechanics. International Journal, 7(5).

[4] Pachoulakis, I., \& Kapetanakis, K. (2012). Augmented reality platforms for virtual fitting rooms. The International Journal of Multimedia \& Its Applications, 4(4), 35.

[5] Erra, U., Scanniello, G., \& Colonnese, V. (2018). Exploring the effectiveness of an augmented reality dressing room. Multimedia Tools and Applications, 77(19), 25077-25107.

[6] Kusumaningsih, A., Kurniawati, A., Angkoso, C. V., Yuniarno, E. M., \& Hariadi, M. (2017, November). User experience measurement on virtual dressing room of Madura batik clothes. In 2017 International Conference on Sustainable Information Engineering and Technology (SIET) (pp. 203-208). IEEE.

[7] Zhang, W., Matsumoto, T., Liu, J., Chu, M., \& Begole, B. (2008, January). An intelligent fitting room using multi-camera perception. In Proceedings of the 13th international conference on Intelligent user interfaces (pp. 60-69).

[8] Peukert, C., Pfeiffer, J., Meißner, M., Pfeiffer, T., \& Weinhardt, C. (2019). Shopping in virtual reality stores: The influence of immersion on system adoption. Journal of Management Information Systems, 36(3), 755-788.

[9] Papagiannidis, S., Pantano, E., See-To, E. W., \& Bourlakis, M. (2013). Modelling the determinants of a simulated experience in a virtual retail store and users' product purchasing intentions. Journal of Marketing Management, 29(13-14), 1462-1492.

[10] Gloor, P., Fischbach, K., Gluesing, J., Riopelle, K., \& Schoder, D. (2018). Creating the collective mind through virtual mirroring based learning. Development and Learning in Organizations: An International Journal.

[11] Bramley, I., Goode, A., Anderson, L., \& Mary, E. (2018). Researching in-store, at home: Using virtual reality within quantitative surveys. International Journal of Market Research, 60(4), 344-351. 